\title{
Src-IL-18 signaling regulates the secretion of atrial natriuretic factor in hypoxic beating rat atria
}

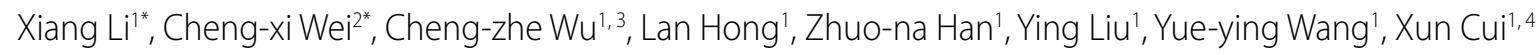

'Department of Physiology, School of Medicine, Yanbian University, Yanji, Jilin, China

${ }^{2}$ Mongolia University For Nationalities, Tongliao, Inner Mongolia, China

${ }^{3}$ Institute of Clinical Medicine, Yanbian University, Yanji, Jilin, China

${ }^{4}$ Cellular Function Research Center, Yanbian University, Yanji, Jilin, China

*Both authors equally contributed to the study

\section{Editorial}

by Nikinmaa,

see p. 947

\section{Correspondence to:}

Prof. Xun Cui, MD, PhD,

Department of Physiology,

School of Medical Sciences,

Yanbian University, No. 977

Gongyuan Road,

Yanji City, Jilin Province,

133002, China

phone: +864332435132,

e-mail: cuixun@ybu.edu.cn

Copyright by the Author(s), 2021

Kardiol Pol. 2021:

79 (9): 972-979.

DOI: 10.33963/KP.a2021.0051

Received:

March 9, 2021

Revision accepted

June 25, 2021

Published online:

June 26, 2021

\section{A B S T R A C T}

Background: Interleukin (IL)-18 is produced mainly in the heart and can be associated with the development of cardiac hypertrophy that leads to cardiac dysfunction. However, the effects of hypoxia on IL-18 expression and atrial natriuretic factor (ANF) secretion remain largely unknown.

Aim: The aim of this study was to assess the effect of hypoxia on IL-18 production and its role in ANF secretion by using an isolated perfused beating rat atrial model.

Methods: The level of ANF in the perfusates was determined by radioimmunoassay, and the protein levels of Src, IL-18 and its receptors (IL-18-Ra and IL-18-R ), Rho guanine nucleotide exchange factor (RhoGEF) and RhoA, activating transcription factor 3 (ATF3), T cell factor (TCF) 3 and 4, and lymphoid enhancer factor (LEF) 1 in atrial tissue samples were detected by Western blotting.

Results: Hypoxia significantly upregulated the expression of the non-receptor tyrosine kinase Src, and this effect was blocked by endothelin-1 receptor type A (BQ123) and type B (BQ788) antagonists. Hypoxia also enhanced the expression of RhoGEF and RhoA concomitantly with the upregulation of IL-18, IL-18-Ra and IL-18-R $\beta$. The hypoxia-induced RhoGEF and RhoA were abolished by Src inhibitor $1(\mathrm{SrCl})$, and the protein levels of IL-18 and its two receptors were also blocked by Srcl. Moreover, the hypoxia-induced expression levels of ATF3, TCF3, TCF4 and LEF1 were repealed by IL-18 binding protein, and the hypoxia-promoted secretion of ANF was also obviously attenuated by this binding protein.

Conclusions: These findings imply that SrC-IL-18 signaling is involved in the release of ANF in hypoxic beating rat atria.

Key words: atrial natriuretic factor, endothelin-1, hypoxia, interleukin-18, non-receptor tyrosine kinase Src

Kardiol Pol 2021; 79, 9: 972-979

\section{INTRODUCTION}

Interleukin (IL)-18 is a proinflammatory cytokine that has been discovered from the serum of mice injected and infected with endotoxin and Mycobacterium bovis bacillus Calmette-Guérin, respectively [1]. Accumulating evidence has demonstrated that endothelial cells, infiltrated neutrophils, smooth muscle cells, resident macrophages, and cardiomyocytes can generate IL-18 in response to infection or injury $[2,3]$. IL-18 is associated with the development of cardiomyocyte hypertrophy that leads to extracellular matrix remodeling and myocardial contractile dysfunction in different animal models of pressure overload, acute myocardial infarction, and lipopolysaccharide-induced dysfunction [4-6]. The overexpression of IL-18 is related to an increased risk of developing cardiovascular diseases and confer a poor prognosis in these patients [3]. Additionally, $\mathrm{IL}-18$ upregulates the protein and mRNA expression of atrial natriuretic peptide (ANF) concomitantly with an increase in the expression of GATA4 through the phosphatidylinositol 3 kinase-Akt pathway [7, 8]. As a peptide hormone, 
WHAT'S NEW?

In this study, hypoxia regulates atrial natriuretic factor (ANF) secretion by activating the Src-interleukin-18-activating transcription factor 3 signaling pathway. These effects were controlled by hypoxia-induced endogenous endothelin-1 (ET-1) via ET receptors in isolated beating rat atria. The findings reveal a new mechanism for the regulation of ANF secretion during hypoxia, which provides a new target for the treatment of myocardial ischemia/hypoxia-related diseases.

ANF is produced and released from the atrial myocytes in response to certain stimuli (e.g., stretch and hypoxia) [9]. It possesses beneficial effects on cardiovascular diseases such as natriuresis, diuresis, vasodilation, blood pressure regulation [10]. ANF secretion is strongly augmented by hypoxia, which can regulate cellular adaptation to hypoxia, protect cardiomyocytes against ischemia/reperfusion injury, decrease the risk of heart failure and prevent ventricular remodeling after dilated cardiomyopathy [11-13]. In our previous work [14], we found that hypoxia could stimulate the secretion of ANF by regulating endogenous endothelin-1 (ET-1) expression through activation of cyclooxygenase 2-lipocalin-type prostaglandin D synthase-peroxisome proliferator-activated receptor $\gamma$ signaling pathway in beating rat atria, but the precise mechanisms by which hypoxia regulates atrial ANF secretion are still unclear. Other studies have demonstrated that the mRNA levels of IL-18 and pro-IL-18 are notably upregulated in the left ventricular myocardium after ischemia/reperfusion in the mouse model [15], while the levels of IL-18 in the circulation and myocardial tissue are also upregulated in heart failure patients $[16,17]$. However, the effects of hypoxia on IL-18 expression and its role in ANF secretion remain largely unknown. This study aims to determine the effects and underlying mechanisms by which hypoxia regulates ANF secretion in beating rat atria.

\section{METHODS}

\section{Reagents}

Human and murine IL-18 binding protein (IL-18-BP) isoforms are active across species [18]. Thus, recombinant human IL-18-BP (100.0 ng/ml; CB99) was selected in this study and was purchased from Novoprotein (Shanghai, China). Endothelin receptor type A (ETA) antagonist BQ123 (0.3 $\mu \mathrm{M}$; $B 150)$ and endothelin receptor type $B$ (ETB) antagonist BQ788 (0.3 $\mu \mathrm{M}$; B157) were supplied by Sigma-Aldrich (St. Louis, MO, USA). Src tyrosine kinase antagonist or Src inhibitor 1 (Srcl, 1.0 MM; HY-101053) and Rhosin hydrochloride (Rhosin) or Rho guanine nucleotide exchange factor (RhoGEF) inhibitor (10.0 $\mu \mathrm{M}$; HY12646) were supplied by MedChemExpress (Monmouth Junction, NJ, USA).

\section{Isolation and perfusion of beating rat atria}

To avoid gender interference, 129 Sprague-Dawley rats of different sexes ( 62 male and 67 female; weight: $250-300$ g; age: 18 weeks) were randomly selected to prepare the perfused beating rat atria. The rats were maintained under specific pathogen-free conditions at Yanbian University (Permit No.: SCXK [Ji] 2011-006) and fed a standard chow diet. All experiments were approved by the Animal Care and Use Committee of Yanbian University and were in accordance with the laboratory animal guidelines of the US National Institutes of Health. The perfused beating left atrium was isolated from each rat and prepared according to the previous methods $[14,19]$. Transmural electrical field stimulation (30-40 V, $0.3 \mathrm{~ms}$ ) was operated at a frequency of $1.5 \mathrm{~Hz}$ using a luminal electrode. To measure the changes in pulse pressure variation, atrial pacing was conducted by perfusing HEPES buffer solution into the atrium via a peristaltic pump $(1.0 \mathrm{ml} / \mathrm{min})$. An adequate amount of oxygen was supplied to the perfused atrium throughout the whole process. The HEPES buffer solution ( $\mathrm{pH}$ 7.4) consisted of $\mathrm{NaCl}, \mathrm{NaHCO}_{3}$, HEPES, glucose, $\mathrm{KCl}$, $\mathrm{CaCl}_{2}, \mathrm{MgCl}_{2}$, and bovine serum albumin $(118,25,10,10$, 4.7, 2.5, $1.2 \mathrm{mmol} / \mathrm{l}$, and $0.1 \%$, respectively).

\section{Construction of the hypoxic atrial model}

To establish a hypoxic atria model, $\mathrm{O}_{2}$ was replaced with $\mathrm{N}_{2}$ by substituting the standard HEPES buffer with an $\mathrm{N}_{2}$-saturated HEPES buffer. The $\mathrm{P}_{\mathrm{O} 2}$ of $\mathrm{N}_{2}$ saturated perfusates was $55 \pm 2 \mathrm{~mm} \mathrm{Hg}$, indicating that the hypoxic condition has reached a severe level.

\section{Determination of ANF levels}

The concentrations of ANF in the perfusates were determined by the lodine [125I] Atrial Natriuretic Factor Radioimmunoassay Kit, according to the previous methods [14, 19]. The inter- and intra-assay coefficients of variation for this assay were $<15 \%$ and $<10 \%$, respectively. The amounts of ANF secretion are presented as $\mathrm{ng} / \mathrm{min} / \mathrm{g}$ of atrial tissue wet weight.

\section{Experimental procedures}

The rats were randomly assigned to 8 groups ( $n=6$ in each group): (1) normoxia, (2) hypoxia, (3) IL-18BP + hypoxia, (4) BQ123 + hypoxia, (5) BQ788 + hypoxia, (6) BQ123 + BQ788 + hypoxia, (7) Srcl + hypoxia, and (8) Rhosin + hypoxia groups.

To stabilize the atrial dynamic parameters, each atrium was subjected to perfusion over a period of 1 hour. Following two 12-minutes normoxia cycles, the perfusates were infused with hypoxic buffer for four cycles. Samples were collected at $4^{\circ} \mathrm{C}$ every 2 minutes to measure ANF levels. After perfusion, the atrial tissues were immediately frozen and kept at $-80^{\circ} \mathrm{C}$. Subsequently, another set of 
experiments was conducted to elucidate the mechanisms of hypoxia-regulated ANF secretion. After one normoxia cycle, one treatment cycle was followed by four infusion cycles of hypoxia plus the treatment agent(s). The normoxia experiments were carried out with the infusion of normoxic buffer for 6 cycles.

\section{Immunoblot analysis}

Each left atrium tissue was rinsed with saline and transferred into RIPA buffer (Solarbio Institute of Biotechnology, Shanghai, China) containing 1.0 M protease inhibitor (Beyotime Biotechnology) and 1.0 M phosphatase inhibitor (Bestbio, Shanghai, China) for homogenization. Immunoblotting, quantitative autoradiography, and densitometric analysis were carried out according to the previous methods [14, 19]. Briefly, the equal amounts $(40 \mu \mathrm{g})$ of protein samples were subjected to SDS-PAGE and subsequently transferred onto PVDF membranes. After blocking (5\% BSA; SW3015; Solarbio) for 2 hours, the membranes were separately incubated with anti-IL-18 (1:000; bs-0529R; Bioss, Beijing, China), anti-IL-18-type a receptor (IL-18-Ra; 1:500; BS9268; Bioworld Technology, Nanjing, China), anti-IL-18-type $\beta$ receptor (IL-18-Rß; 1:1000; bs-2616R; Bioss), anti-Src (1:500; 04-889; Millipore, MA, US), anti-p115RhoGEF (1:1000;
BS5901; Bioworld Technology), anti-RhoA (1:1000; 107491-AP; Proteintech, Wuhan, China), anti-activating transcription factor 3 (ATF3; 1:1000; DF6660; Affinity, Changzhou, China), anti-T cell factor (TCF) 3 (1:500; DF4573; Affinity), anti-TCF4 (1:500; DF7622; Affinity), anti-lymphoid enhancer factor 1 (LEF1; 1:1000; DF7570; Affinity), or anti- $\beta$-actin antibodies (1:1000; AP0060; Bioworld Technology) overnight at $4^{\circ} \mathrm{C}$. The membranes were then washed and incubated again with a secondary antibody (1:3000; AP132P; Nachuan biotech, Changchun, China) for 1.5 hours at room temperature. Visualization of the protein bands was performed using ECL Western blotting substrate kit (Raybiotech, Atlanta, GA, USA) and a chemiluminescent detection system. Densitometric analysis of the protein blots was conducted using ImageJ software (NIH, Bethesda, MA, USA).

\section{Statistical analysis}

All data were normally distributed(Kolmogorov-Smirnov test) and presented as mean (standard error of the mean, SEM). IBM SPSS Statistics software ver. 19.0 (IBM Corp, Armonk, NY, USA) and GraphPad Prism ver. 9.1.2 software (San Diego, CA, USA) were employed for statistical analyses. The Student's t-test was used to compare the difference between two groups (Supplementary material, Figure S1B), while one-way
A

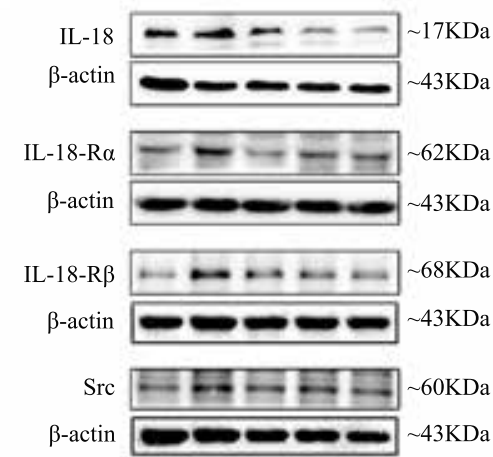

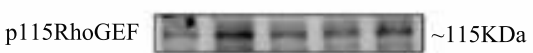
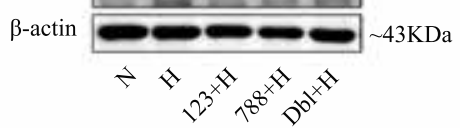

\section{B}
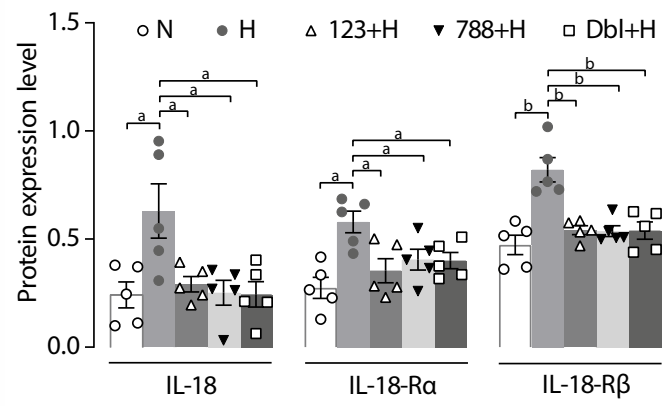

IL-18-R $\beta$

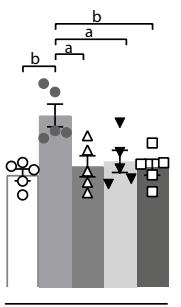

Src

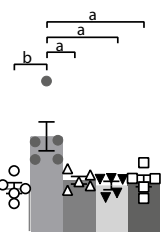

p115RhoGEF

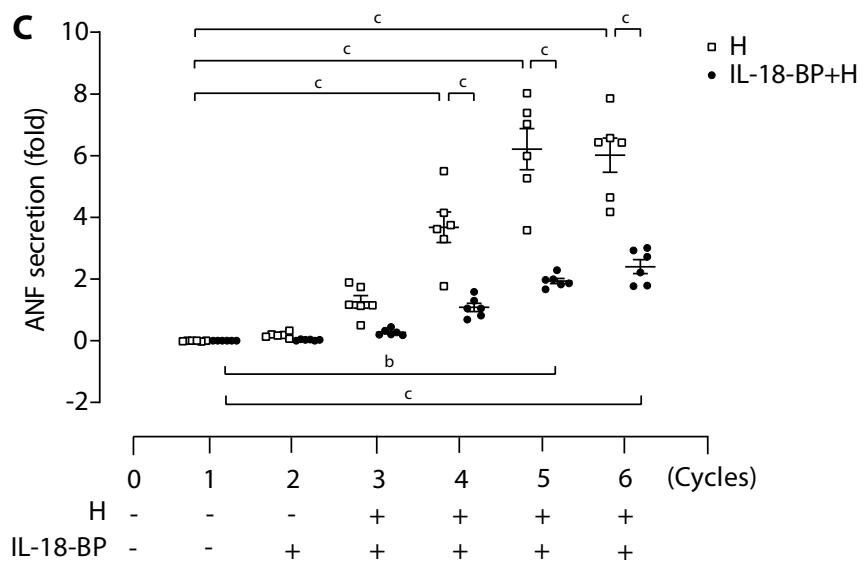

Figure 1. Effects of hypoxia on IL-18, IL-18-Ra, IL-18-R $\beta$, Src, and RhoGEF expression, and ANF secretion in isolated beating rat atria. A. Representative Western blot bands. B. Quantification of protein levels ( $n=5,3$ male and 2 female/per group). C. Changes of ANF secretion (the values were represented the averages of each cycle, $\mathrm{n}=6,3$ male and 3 female/per group). Data were expressed as mean (SEM). ${ }^{\mathrm{a}} P<0.05 ;{ }^{\mathrm{b}} P$ $<0.01$; $P<0.0001$. IL-18-BP, and hypoxia, respectively

Abbreviations: 123, BQ123, an ETA antagonist; 788, BQ788, an ETB antagonist; ANF, atrial natriuretic factor; Dbl, BQ123+BQ788; $\mathrm{H}$, hypoxia; IL-18, interleukin-18; IL-18-BP, IL-18 binding protein, an IL-18 antagonist; IL-18-Ra, IL-18-type a receptor; IL-18-R $\beta$, IL-18-type $\beta$ receptor; N, normoxia; RhoGEF, Rho guanine nucleotide exchange factor 
ANOVA followed by Dunnett's multiple comparison (Figures $1 B, 2 B, 3 B, 4 B$, and Supplementary material, Figure S2B). Two-way ANOVA followed by Bonferroni post hoc test was used for Figure 1C, 2C, and 3C. $P$-value $<0.05$ was considered statistically significant. The Bonferroni-corrected $P$-value thresholds were calculated as follows: for the study of associations between normoxia, hypoxia, and hypoxia plus the treatment agent (hypoxia + IL-18-BP, hypoxia + Srcl, hypoxia + Rhosin), a significance level of $a=0.05$, so the corrected $P$-value threshold was $0.05 / 3=0.0167$.

\section{RESULTS}

\section{Effects of hypoxia on IL-18 expression and ANF secretion}

To explore the roles of hypoxia in regulating IL-18 expression and ANF secretion, a series of experiments were conducted using the isolated atrial perfusates and tissue of a hypoxic rat model. The results demonstrated that hypoxia remarkably upregulated the expression of IL-18 ( $P=0.02$ vs normoxia) and its type a (IL-18-Ra) and $\beta$ (IL-18-R $\beta$ ) receptors ( $P=0.03,0.001$ vs normoxia, respectively, Supplementary material, Figure $S 1 A)$. Corrected $P$-values were applied to multiple comparisons of ANF secretion using Bonferroni posthoc analysis. Posthoc analysis showed that hypoxia also significant increase
ANF secretion ( $P<0.0001$ vs normoxia; Figure $1 C)$, and the ANF secretion was obviously decreased by IL-18-BP, an IL18 antagonist $(P<0.0001$ vs hypoxia; Figure $1 C)$, reaching the Bonferroni-corrected $P$-value threshold $(<0.0167)$. In addition, the upregulated expression levels of IL-18 and its receptors, IL-18-Ra and IL-18-R $\beta$, induced by hypoxia were completely suppressed by BQ123 $(P=0.03,0.02$, 0.002 vs hypoxia) and $B Q 788(P=0.03,0.04,0.002$ vs hypoxia), the ETA and ETB antagonists (Figure 1A, 1B). These findings indicated that IL-18 altered by endogenous ET-1 was involved in the release of ANF in beating rat hypoxic atria.

\section{Effects of hypoxia on the expression of Src and RhoA as well as the secretion of ANF}

In accordance with the role of Src in inflammatory actions and the relationship between RhoA and interleukins, we determined the effects of hypoxia on the protein levels of Src, RhoA, as well as the secretion of ANF. The results indicated that hypoxia markedly upregulated the expression of $\operatorname{Src}(P=0.002$ vs normoxia; Figure $1 \mathrm{~A}, 1 \mathrm{~B})$, but such upregulation was entirely abolished by $B Q 123(P=0.01$ vs hypoxia) and BQ788 ( $P=0.02$ vs hypoxia; Figure $1 \mathrm{~A}, 1 \mathrm{~B})$. Hypoxia was also noticeably upregulated the expression of p115RhoGEF ( $P=0.01$ vs normoxia; Figure $1 \mathrm{~A}, 1 \mathrm{~B})$ and RhoA ( $P=0.005$ vs normoxia; Figure $2 \mathrm{~A}, 2 \mathrm{~B})$. However,

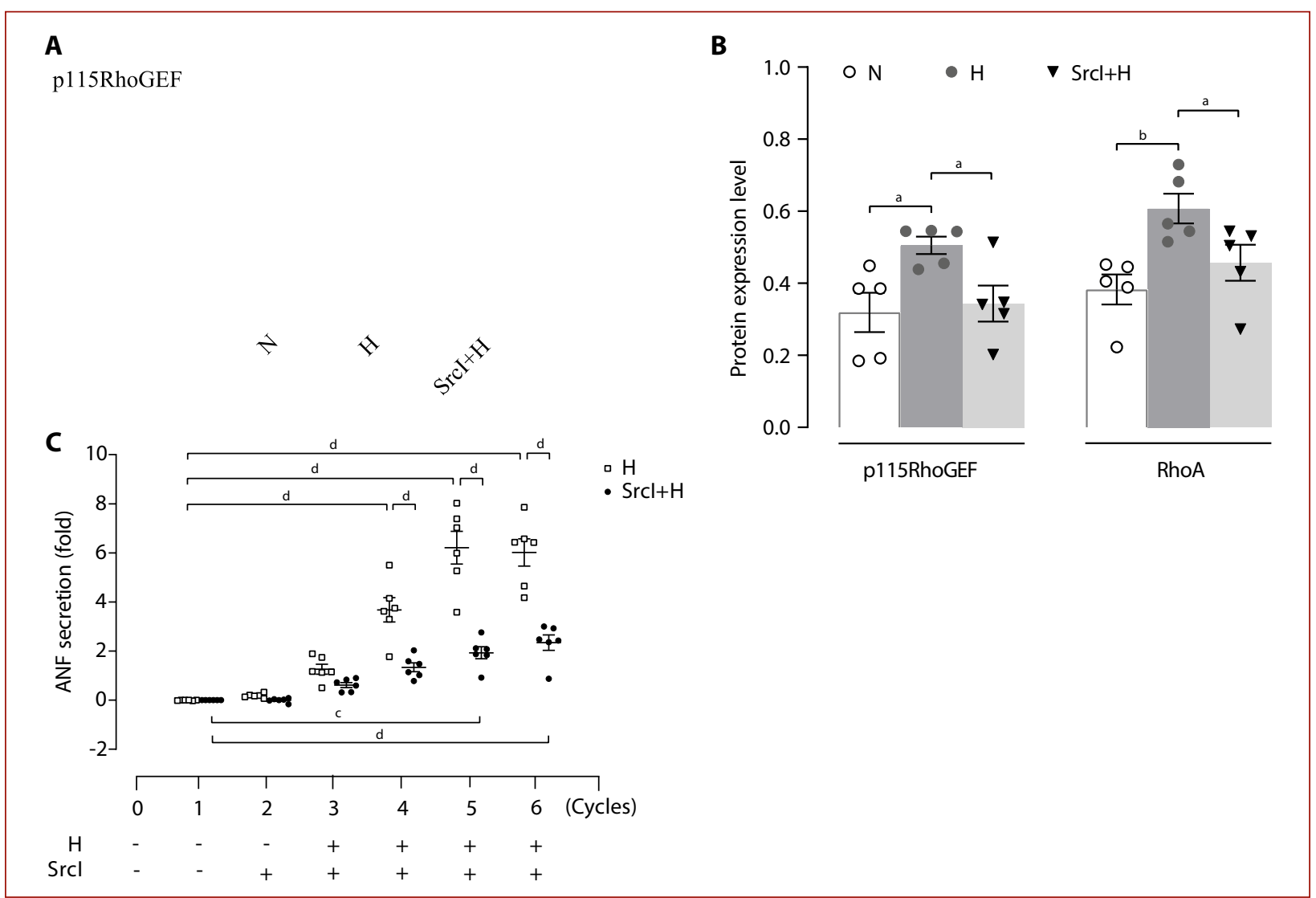

Figure 2. Effects of Src on RhoGEF and RhoA protein levels as well as the secretion of ANF in beating rat atria. A. Representative Western blot bands. B. Quantification of protein levels ( $n=5,3$ male and 2 female/per group). C. Changes of ANF secretion ( $n=6,3$ male and 3 female/per group). Data were expressed as mean (SEM). ${ }^{a} P<0.05 ;{ }^{b} P<0.01 ;{ }^{c}, d p<0.0001$

Abbreviations: Srcl, Src inhibitor 1, a Src antagonist. Other — see Figure 1 
A

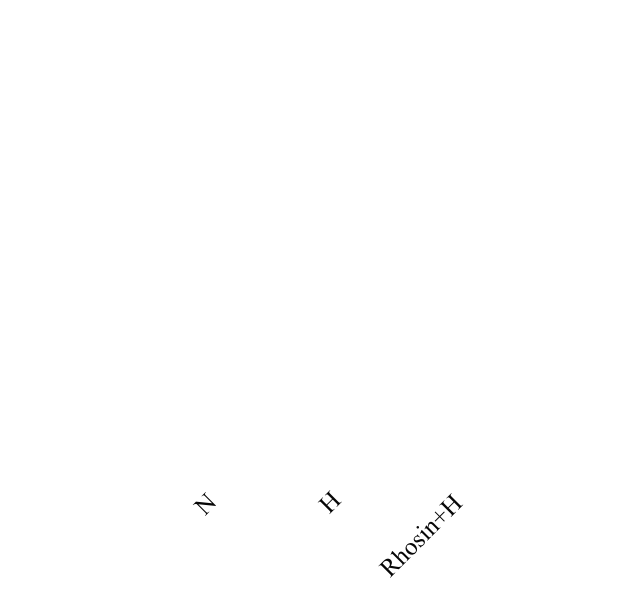

B

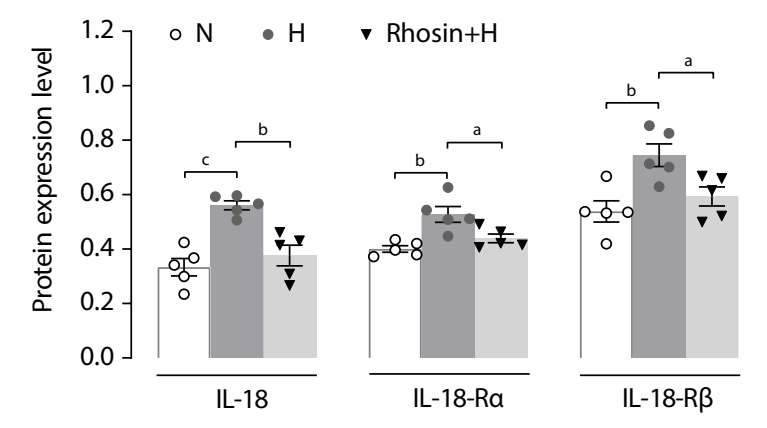

C

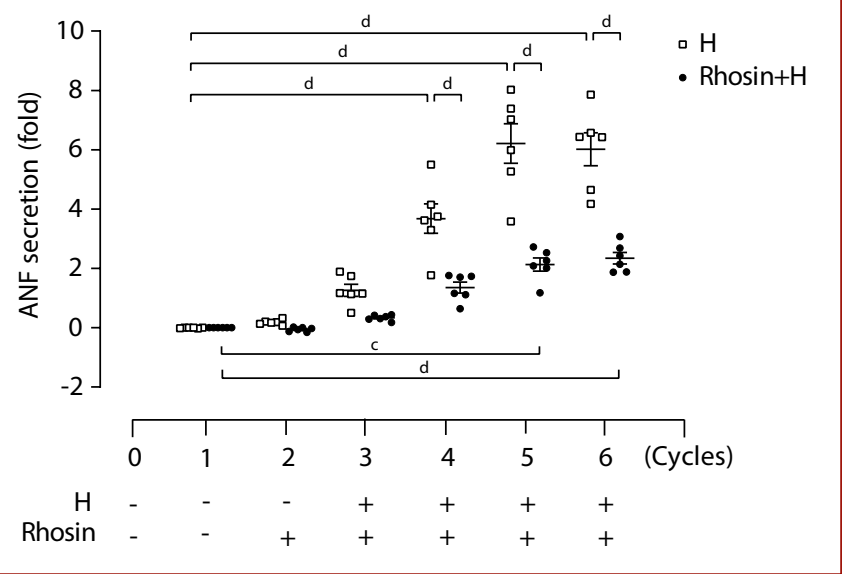

Figure 3. Effects of RhoGEF on the protein levels of IL-18 and its receptors, and the secretion of ANF in hypoxic beating rat atria. A. Representative Western blot bands. B. Quantification of protein levels $(n=5,2$ male and 3 female/per group). C. Changes of ANF secretion ( $n=6$, 3 male and 3 female/per group). Data were expressed as mean (SEM). ${ }^{a} P<0.05 ;{ }^{b} P<0.01 ;{ }^{c} P<0.001 ;{ }^{d} P<0.0001$

Abbreviations: Rhosin, a RhoGEF antagonist. Other — see Figure 1

the hypoxia-induced expression of p115RhoGEF was obviously reduced by $\mathrm{BQ} 123(P=0.02$ vs hypoxia) and BQ788 ( $P=0.01$ vs hypoxia; Figure 1A, 1B). Moreover, a Src antagonist Srcl also downregulated the expression of p115RhoGEF ( $P=0.02$ vs normoxia) and RhoA $(P=0.049$ vs normoxia; Figure $2 \mathrm{~A}, 2 \mathrm{~B})$. Moreover, posthoc analysis showed that the hypoxia-induced ANF secretion $(P<0.0001$ vs normoxia; Figure $2 C, 3 C)$ was remarkably decreased by $\operatorname{Srcl}(P<0.0001$ vs hypoxia; Figure $2 C)$ and Rhosin, a RhoGEF inhibitor $(P<0.0001$ vs hypoxia; Figure $3 C)$, reaching the Bonferroni-corrected $P$-value threshold $(<0.0167)$. These results demonstrated that Src induced by hypoxia-induced ET-1 could regulate the expression of RhoA through p115RhoGEF activation and mediate the hypoxia-induced release of ANF.

\section{Effects of RhoA on hypoxia-induced expression of IL-18}

To elucidate the role of RhoA in modulating the hypoxia-induced expression of IL-18 and its receptors, a series of experiments were performed using Rhosin. The hypoxia-induced upregulation of IL-18 $(P=0.0002$ vs normoxia) and its receptors (IL-18-Ra and IL-18-R $\beta, P=0.004,0.007$ vs normoxia) were utterly blocked by Rhosin ( $P=0.002,0.03$, 0.02 vs hypoxia, respectively; Figure $3 \mathrm{~A}, 3 \mathrm{~B}$ ). These findings showed that RhoA controlled by Src could regulate the protein levels of IL-18, IL-18-Ra, and IL-18-R $\beta$ under hypoxic conditions.

\section{Effects of IL-18 on hypoxia-induced expression of TCF/LEF}

To understand the mechanisms by which IL-18 mediates hypoxia-induced ANF secretion, the effect of IL-18 on TCF/LEF expression was determined using IL-18-BP. The results demonstrated that hypoxia markedly increased the protein levels of ATF3, TCF3, TCF4, and LEF1 $(P=0.01$, $0.001,0.03,0.005$ vs normoxia, respectively; Figure $4 \mathrm{~A}$ and $4 B)$, but such upregulation patterns were entirely abolished by BQ123 ( $P=0.04,0.03,0.04,0.02$ vs hypoxia) and BQ788 $(P=0.02,0.02,0.03,0.02$ vs hypoxia; Supplementary material, Figure S1A, S1B). IL-18-BP also dramatically suppressed the hypoxia-induced expression of ATF $3(P=0.048$ vs hypoxia), TCF3 ( $P<0.001$ vs hypoxia), TCF4 ( $P=0.04$ vs hypoxia) and LEF1 ( $P=0.04$ vs hypoxia; Figure $4 A, 4 B)$. These findings revealed that IL-18 could regulate the expression of ATF3,TCF3/LEF1, and TCF4/LEF1 in hypoxic beating rat atria. 


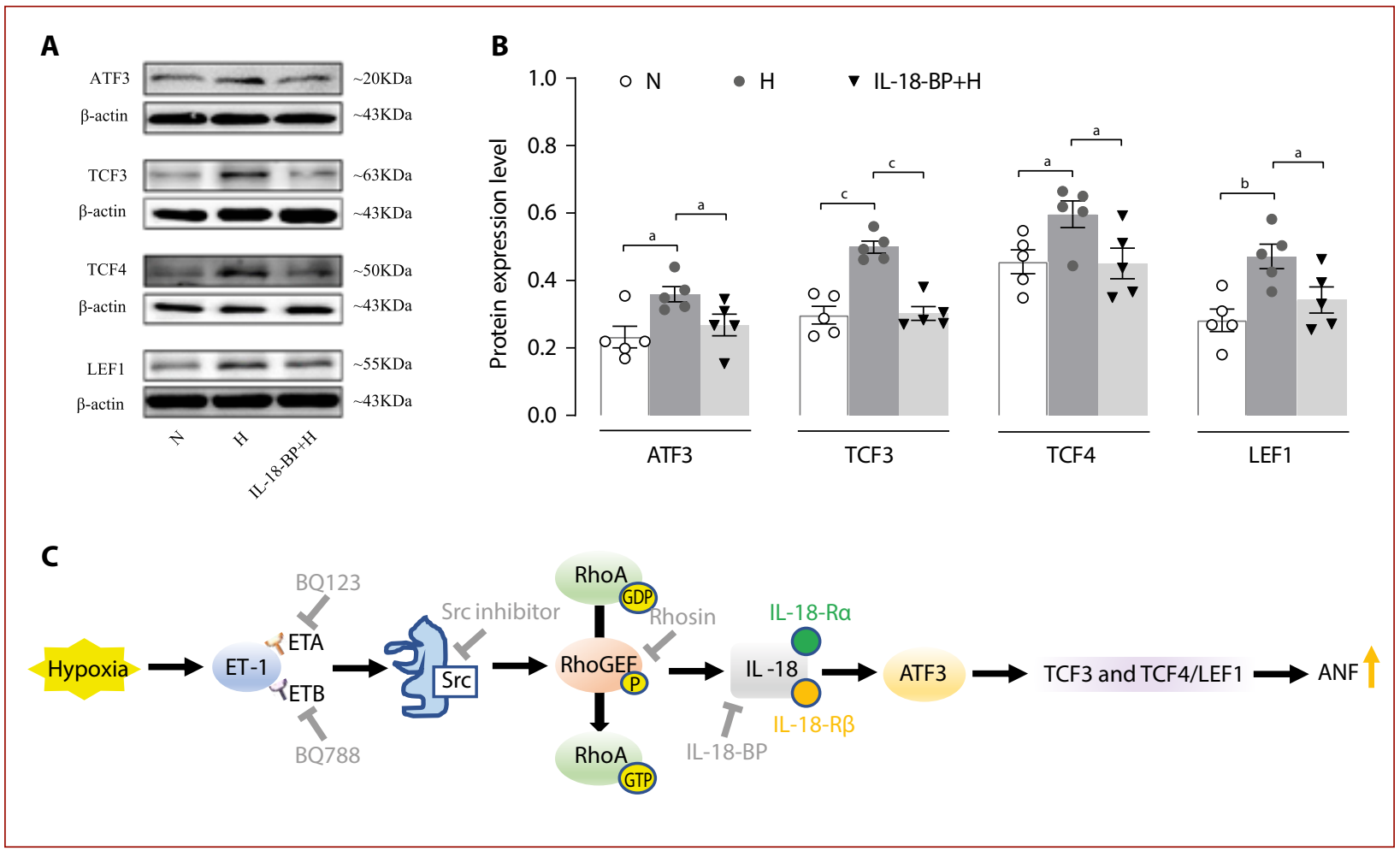

Figure 4. A, B. Effect of IL-18 on the protein levels of ATF3, TCF3, TCF4 and LEF1. C. Schematic mechanisms by which Src-IL-18 regulates ANF secretion during hypoxia. Representative Western blot bands and their quantification ( $n=5,2$ male and 3 female/per group) were presented at $\mathbf{A}$ and $\mathbf{B}$ panels. Data were expressed as mean (SEM). ${ }^{\mathrm{a} P}<0.05 ;{ }^{\mathrm{b}} P<0.01 ;{ }^{\mathrm{c}} P<0.001$

Abbreviations: ATF3, Activating transcription factor 3; TCF3, T cell factor 3; TCF4, T cell factor 4; LEF1, lymphoid enhancer factor 1

\section{DISCUSSION}

This study revealed that hypoxia upregulated the expression of non-receptor tyrosine kinase Src, which obviously increased the expression of IL-18 and its two receptors through activation of RhoA signaling. The increase in IL-18 subsequently upregulated the expression of ATF3, TCF3/LEF1, and TCF4/LEF1, which ultimately regulated hypoxia-induced ANF secretion. These effects were controlled by hypoxia-stimulated ET-1 expression via ET receptors in the isolated beating rat atria (Figure 4C).

Src, a non-receptor tyrosine kinase, is activated by hypoxia and plays a crucial role in triggering intracellular signaling cascades in cardiac myocytes. Additionally, Src is also involved in the processes of cardiovascular disorders induced by ET-1 [20]. Our results demonstrated that hypoxia remarkably upregulated the expression of Src concomitantly with a potent promotion of ANF secretion, while the expression of Src was completely abolished by ET receptor antagonists (BQ123 and BQ788). Moreover, hypoxia-induced ANF secretion was obviously attenuated by Srcl. These data indicated that Src controlled by ET-1 was involved in the atrial secretion of ANF under hypoxic conditions. These results were similar to the previous studies mentioned above and supported a previous study in which the increased Src activity in response to ET-1 treatment was involved in ET-dependent pathway activation of ANF promoter in cardiomyocytes [21].
Rho-kinase is an important downstream effector of RhoA and its activity often requires prior activation of RhoGEFs and subsequently enabling the activation of RhoA [22]. RhoA/Rho-kinase pathway plays a vital role in diverse cellular functions, while its excessive activity is involved in the development of cardiovascular diseases [23]. Results of this study further showed that p115RhoGEF and RhoA were markedly upregulated by hypoxia concomitantly with increased expression of IL-18 and its two receptors. Interestingly, the hypoxia-induced expression of $\mathrm{p} 115 \mathrm{RhoGEF}$ was completely blocked by BQ123, BQ788, and Srcl, whereas Rhosin, an antagonist of RhoGEF, was entirely suppressed the expression of IL-18 and its two receptors. These results imply that Src controlled by endogenous ET-1 via its both antagonists could upregulate the protein levels of IL-18 and its receptors through activation of RhoA, which support a previous study showing that hypoxia- or G-protein-coupled receptor-induced ROS-dependent activation of Src led to the overexpression of RhoA via p115RhoGEF in rat intrapulmonary artery [24]. Moreover, our findings are also consistent with a previous report indicating that aldosterone-induced ET-1 upregulated the expression of IL-18 through activation of Rho-kinase via ETA in cultured rat neonatal cardiomyocytes [25]. However, the effect of ETB on IL-18 expression in this study is controversial with the earlier mentioned reports. This may be, at least in part, explained by the differences in experimental design, sub- 
jects and conditions. However, evidence has implicated the potential role of EdnrB (ETB gene) in cardiovascular function, where this gene could be targeted by pharmacological agents under hypoxic conditions [26]. Additionally, EdnrB ${ }^{-/+}$ heterozygote mice could tolerate different levels of hypoxia by stabilizing cardiac function and maintaining cardiac energy balance [27]. Data from this study also showed that an antagonist of ETB abolished the hypoxia-induced expression of IL-18, IL-18-Ra, and IL-18-R $\beta$, suggesting that it might be beneficial to alleviate inflammation and improve atrial tolerance to hypoxia.

TCF/LEF proteins act as the major downstream effectors of Wnt signaling and can be activated by ATF3, an adaptive-response gene in ATF/cyclic adenosine monophosphate responsive element-binding protein subfamily [28]. It has been shown that TCF/LEF1 binds directly to the ANF promoter via its binding site, thereby regulating ANF transcription in phenylephrine-induced hypertrophic rat cardiomyocytes [29]. Similarly, this study also confirmed that hypoxia dramatically increased the protein levels of ATF3 and TCF3/LEF1 as well as TCF4/LEF1, and such expression patterns were completely blocked by BQ123 and BQ788 concomitantly with an obvious attenuation of ANF secretion. Besides, IL-18-BP mimicked the effects of ET receptor antagonists on the expression of these transcription factors and hypoxia-induced secretion of ANF. Therefore, this study suggests that IL-18 regulated by ET1 -Src is involved in the atrial secretion of ANF in hypoxia beating rat atria through ATF3 as well as TCF3/LEF1 and TCF4/LEF1 signaling.

In summary, the increased expression of Src controlled by hypoxia-induced endogenous ET-1 could lead to the upregulated expression of IL-18 and its receptors through activation of RhoA, which ultimately regulate the atrial secretion of ANF via ATF3 as well as TCF3/LEF1 and TCF4/LEF1 signaling in hypoxic beating rat atria. The increase in ANF secretion during hypoxia might be one of the mechanisms for the inhibition of ET-1 and its downstream IL-18 signaling and exert a beneficial action on cardiovascular health.

\section{Supplementary material}

Supplementary material is available at https://journals. viamedica.pl/kardiologia_polska.

\section{Article information}

Acknowledgment: We would like to express our sincere appreciation to EditSprings (www.editsprings.com) for providing expert linguistic services.

\section{Conflict of interest: None declared.}

Funding: This research was funded by the National Natural Science Foundation of China (Numbers: 81660089 and 81960099).

Open access: This article is available in open access under Creative Common Attribution-Non-Commercial-No Derivatives 4.0 International (CC BY-NC-ND 4.0) license, allowing to download articles and share them with others as long as they credit the authors and the publisher, but without permission to change them in any way or use them commercially. For commercial use, please contact the journal office at kardiologiapolska@ptkardio.pl.

How to cite: Li X, Wei C, Wu C, et al. SrC-IL-18 signaling regulates the secretion of atrial natriuretic factor in hypoxic beating rat atria. Kardiol Pol. 2021; 79(9): 972-979, doi: 10.33963/KP.a2021.0051.

\section{REFERENCES}

1. Nakamura $\mathrm{K}$, Okamura $\mathrm{H}$, Wada $\mathrm{M}$, et al. Endotoxin-induced serum factor that stimulates gamma interferon production. Infect Immun. 1989; 57(2): 590-595, doi: 10.1128/iai.57.2.590-595.1989, indexed in Pubmed: 2492265.

2. Wang M, Markel TA, Meldrum DR. Interleukin 18 in the heart. Shock. 2008; 30(1): 3-10, doi: 10.1097/SHK.0b013e318160f215, indexed in Pubmed: 18562922.

3. O'Brien LC, Mezzaroma E, Van Tassell BW, et al. Interleukin-18 as a therapeutic target in acute myocardial infarction and heart failure. Mol Med. 2014; 20: 221-229, doi: 10.2119/molmed.2014.00034, indexed in Pubmed: 24804827

4. Yu Q, Vazquez R, Khojeini EV, et al. IL-18 induction of osteopontin mediates cardiac fibrosis and diastolic dysfunction in mice. Am J Physiol Heart Circ Physiol. 2009; 297(1): H76-H85, doi: 10.1152/ajpheart.01285.2008, indexed in Pubmed: 19429811.

5. Bellora F, Castriconi R, Doni A, et al. M-CSF induces the expression of a membrane-bound form of IL-18 in a subset of human monocytes differentiating in vitro toward macrophages. Eur J Immunol. 2012; 42(6): 1618-1626, doi: 10.1002/eji.201142173, indexed in Pubmed: 22678914.

6. Takahashi M. NLRP3 inflammasome as a novel player in myocardial infarction. Int Heart J. 2014; 55(2): 101-105, doi: 10.1536/ihj.13-388, indexed in Pubmed: 24632952.

7. Chandrasekar B, Mummidi S, Claycomb WC, et al. Interleukin-18 is a pro-hypertrophic cytokine that acts through a phosphatidylinositol 3-kinase-phosphoinositide-dependent kinase-1-Akt-GATA4 signaling pathway in cardiomyocytes. J Biol Chem. 2005; 280(6): 4553-4567, doi: 10.1074/jbc.M411787200, indexed in Pubmed: 15574430.

8. Colston JT, Boylston WH, Feldman MD, et al. Interleukin-18 knockout mice display maladaptive cardiac hypertrophy in response to pressure overload. Biochem Biophys Res Commun. 2007; 354(2): 552-558, doi: 10.1016/j.bbrc.2007.01.030, indexed in Pubmed: 17250807.

9. Cho KW, Seul KH, Ryu H, et al. Characteristics of distension-induced release of immunoreactive atrial natriuretic peptide in isolated perfused rabbit atria. Regul Pept. 1988;22(4):333-345, doi: 10.1016/0167-0115(88)901103, indexed in Pubmed: 2973090.

10. McGrath MF, de Bold ML, de Bold AJ. The endocrine function of the heart. Trends Endocrinol Metab. 2005; 16(10): 469-477, doi: 10.1016/j. tem.2005.10.007, indexed in Pubmed: 16269246.

11. Arjamaa $O$, Nikinmaa $M$. Hypoxia regulates the natriuretic peptide system. Int J Physiol Pathophysiol Pharmacol. 2011; 3(3): 191-201, indexed in Pubmed: 21941610.

12. Hong $\mathrm{L}, \mathrm{Xi}$ J, Zhang $\mathrm{Y}$, et al. Atrial natriuretic peptide prevents the mitochondrial permeability transition pore opening by inactivating glycogen synthase kinase $3 \beta$ via PKG and PI3K in cardiac H9c2 cells. Eur J Pharmacol. 2012; 695(1-3): 13-19, doi: 10.1016/j.ejphar.2012.07.053, indexed in Pubmed: 22975711.

13. Wang D, Gladysheva IP, Fan THM, et al. Atrial natriuretic peptide affects cardiac remodeling, function, heart failure, and survival in a mouse model of dilated cardiomyopathy. Hypertension. 2014; 63(3): 514-519, doi: 10.1161/HYPERTENSIONAHA.113.02164, indexed in Pubmed: 24379183.

14. Li X, Han ZN, Liu Y, et al. Endogenous ET-1 promotes ANP secretion through activation of COX2-L-PGDS-PPARY signaling in hypoxic beating rat atria. Peptides. 2019; 122: 170150, doi: 10.1016/j.peptides.2019.170150, indexed in Pubmed: 31541683.

15. Woldbaek PR. Increased cardiac IL-18 mRNA, pro-IL-18 and plasma IL-18 after myocardial infarction in the mouse; a potential role in cardiac dysfunction. Cardiovascular Research. 2003; 59(1): 122-131, doi: 10.1016/s0008-6363(03)00339-0.

16. Oberholzer A, Steckholzer U, Kurimoto M, et al. Interleukin-18 plasma levels are increased in patients with sepsis compared to severely injured patients. Shock. 2001; 16(6):411-414, doi: 10.1097/00024382-20011606000001, indexed in Pubmed: 11770036. 
17. Naito Y, Tsujino T, Fujioka Y, et al. Increased circulating interleukin-18 in patients with congestive heart failure. Heart. 2002; 88(3): 296-297, doi: 10.1136/heart.88.3.296, indexed in Pubmed: 12181230.

18. Banda NK, Vondracek A, Kraus D, et al. Mechanisms of inhibition of collagen-induced arthritis by murine IL-18 binding protein. J Immunol. 2003; 170(4): 2100-2105, doi: 10.4049/jimmunol.170.4.2100, indexed in Pubmed: 12574381.

19. Li X, Zhang Y, Zhang Bo, et al. HIF-1a-I-PGDS-PPARy regulates hypoxia-induced ANP secretion in beating rat atria. Prostaglandins Other Lipid Mediat. 2018; 134: 38-46, doi: 10.1016/j.prostaglandins.2017.12.001, indexed in Pubmed: 29287795.

20. Seko Y, Takahashi N, Tobe K, et al. Hypoxia and hypoxia/reoxygenation activate Src family tyrosine kinases and p21ras in cultured rat cardiac myocytes. Biochem Biophys Res Commun. 1996; 226(2): 530-535, doi: 10.1006/bbrc.1996.1389, indexed in Pubmed: 8806668.

21. Kovacic B, llić D, Damsky CH, et al. c-Src activation plays a role in endothelin-dependent hypertrophy of the cardiac myocyte. J Biol Chem. 1998; 273(52): 35185-35193, doi: 10.1074/jbc.273.52.35185, indexed in Pubmed: 9857056.

22. Jaiswal M, Gremer L, Dvorsky R, et al. Mechanistic insights into specificity, activity, and regulatory elements of the regulator of $\mathrm{G}$-protein signaling (RGS)-containing Rho-specific guanine nucleotide exchange factors (GEFs) p115, PDZ-RhoGEF (PRG), and leukemia-associated RhoGEF (LARG). J Biol Chem. 2011; 286(20): 18202-18212, doi: 10.1074/jbc.M111.226431, indexed in Pubmed: 21454492.

23. Shimokawa H, Sunamura S, Satoh K. RhoA/Rho-Kinase in the cardiovascular system. Circ Res. 2016; 118(2): 352-366, doi: 10.1161/CIRCRESAHA.115.306532, indexed in Pubmed: 26838319.
24. MacKay CE, Shaifta Y, Snetkov V, et al. ROS-dependent activation of RhoA/Rho-kinase in pulmonary artery: Role of Src-family kinases and ARHGEF1. Free Radic Biol Med. 2017; 110: 316-331, doi: 10.1016/j.freeradbiomed.2017.06.022, indexed in Pubmed: 28673614.

25. Doi T, Sakoda T, Akagami T, et al. Aldosterone induces interleukin-18 through endothelin-1, angiotensin II, Rho/Rho-kinase, and PPARs in cardiomyocytes. Am J Physiol Heart Circ Physiol. 2008; 295(3): H1279-H1287, doi: 10.1152/ajpheart.00148.2008, indexed in Pubmed: 18660453.

26. Green DS, Rupasinghe $C$, Warburton $R$, et al. A cell permeable peptide targeting the intracellular loop 2 of endothelin $B$ receptor reduces pulmonary hypertension in a hypoxic rat model. PLoS One. 2013; 8(11): e81309, doi: 10.1371/journal.pone.0081309, indexed in Pubmed: 24312288.

27. Stobdan T, Zhou D, Ao-leong E, et al. Endothelin receptor B, a candidate gene from human studies at high altitude, improves cardiac tolerance to hypoxia in genetically engineered heterozygote mice. Proc Natl Acad Sci U S A. 2015; 112(33): 10425-10430, doi: 10.1073/pnas.1507486112, indexed in Pubmed: 26240367.

28. Hrckulak D, Kolar M, Strnad H, et al. TCF/LEF transcription factors: an update from the internet resources. Cancers (Basel). 2016; 8(7), doi: 10.3390/cancers8070070, indexed in Pubmed: 27447672.

29. Zhang CG, Jia ZQ, Li BH, et al. Beta-Catenin/TCF/LEF1 can directly regulate phenylephrine-induced cell hypertrophy and Anf transcription in cardiomyocytes. Biochem Biophys Res Commun. 2009; 390(2): 258-262, doi: 10.1016/j.bbrc.2009.09.101, indexed in Pubmed: 19799869. 